\title{
Experimental and numerical analyses of adhesively bonded T-joints under crash loading
}

\author{
M. May and H. Voß \\ Fraunhofer Institute for Highspeed Dynamics, EMI, Eckerstr. 4, 79104 Freiburg, Germany
}

\begin{abstract}
Adhesively bonded metallic T-joints were subjected to impact at different loading directions and loading rates ranging from quasi-static to crash. Different damage and failure mechanisms were observed depending on the loading configuration. Finite element simulations were performed with ABAQUS/explicit using a user-defined, fully rate dependent material model for cohesive elements describing the material response of the adhesive bond lines. The FE simulation results were in good agreement with the experimental evidence, capturing both, the observed damage mechanisms, and the recorded force-displacement response.
\end{abstract}

\section{Introduction}

In recent years, developments in the automotive industry have been driven by the need for a reduction of fuel consumption and $\mathrm{CO}_{2}$ emissions by reduction of the vehicle weight. At the same time, the crashworthiness of the vehicle had to be maintained. This was achieved by introducing high-strength steels and new joining techniques such as adhesive bonding. However, the response of adhesively bonded metallic joints under crash-loading is not yet fully understood due to the sheer complexity of the problem. Factors affecting the response of such joints are, for example, the elastic and plastic deformation of the adhesive and the adherends, geometry (e.g. bond line thickness, overlap length), surface preparation during manufacturing, and loading rate. In this paper we present experimental and numerical data on adhesively bonded T-joints subjected to different loading rates and loading directions.

\section{Experimental analysis}

\subsection{Joint geometry}

Several experiments have been conducted in a previous project [1] using adhesively bonded T-joints consisting of two cap profiles bonded together. The drawback of such setup is that a side impact can only be realized by adding additional loading blocks to the upper cap profile. However, when performing dynamic tests adding additional dead mass into the system is not recommended as inertia effects may have significant influence on the loads recorded during the test. Additionally, the loads were introduced differently under quasi-static and dynamic loading conditions - a real comparison of static and dynamic tests was therefore not possible. Here, we designed a new T-joint geometry addressing these issues, thus allowing testing in multiple directions at different loading rates without adding any additional masses into the system. The approach taken was to bond a rectangular profile onto a cap profile acting as base (see Fig. 1). The T-joints were manufactured at Fraunhofer IFAM in

a e-mail: michael.may@emi .fraunhofer.de
Bremen. The T-joints are of length $300 \mathrm{~mm}$ and height $202 \mathrm{~mm}$. The top hat profile is $50 \mathrm{~mm}$ wide, the rectangular profile is of dimension $48.3 \mathrm{~mm} \times 49.5 \mathrm{~mm}$ (including the back sheet). The adherends were made of Thyssen Krupp DP K 30/50 steel sheets of thickness $t=1.2 \mathrm{~mm}$. The sheet surfaces were activated by plasma treatment in order to ensure cohesive bond line failure rather than adhesive bond line failure. The adhesive used for bonding of the steel sheets was Dow BETAMATE $1496 \mathrm{~V}$, a crash optimized adhesive for automotive applications. The thickness of the bond lines was adjusted to $0.3 \mathrm{~mm}$ using glass spheres.

\subsection{Experimental setup}

The T-joints were mounted in a servo-hydraulic test machine allowing loading rates up to $5 \mathrm{~m} / \mathrm{s}$ as shown in Fig. 2. The ends of the cap profile were fixed in a steel rig mounted onto a steel frame. Front and side impact loading of the adhesively bonded metallic T-joint can be realized by a $90^{\circ}$ rotation of the rig. A lightweight aluminium impactor was chosen for reduction of noise in the load signal. The impactor was instrumented with a dynamic $60 \mathrm{kN}$ load cell and an acceleration sensor. All tests were recorded with high-speed cameras allowing detailed analyses of the damage and failure sequences occurring during the tests. The experiments were performed at three different loading rates: $0.5 \mathrm{~mm} / \mathrm{s}, 50 \mathrm{~mm} / \mathrm{s}$, and $4800 \mathrm{~mm} / \mathrm{s}$. At least four T-joints were tested for each loading rate and direction.

\subsection{Results}

\subsubsection{Front impact}

The force-displacement curves recorded during front impact loading are summarized in Fig. 3. High speed video footage of the typical failure sequence observed during the experiments is given in Fig. 4. The upper diagram compares quasi-static (red) and medium dynamic (green, dashed) results, the lower diagram shows forcedisplacement curves recorded during crash loading. For quasi-static and medium dynamic loading, the forcedisplacement curves feature a characteristic peak load 


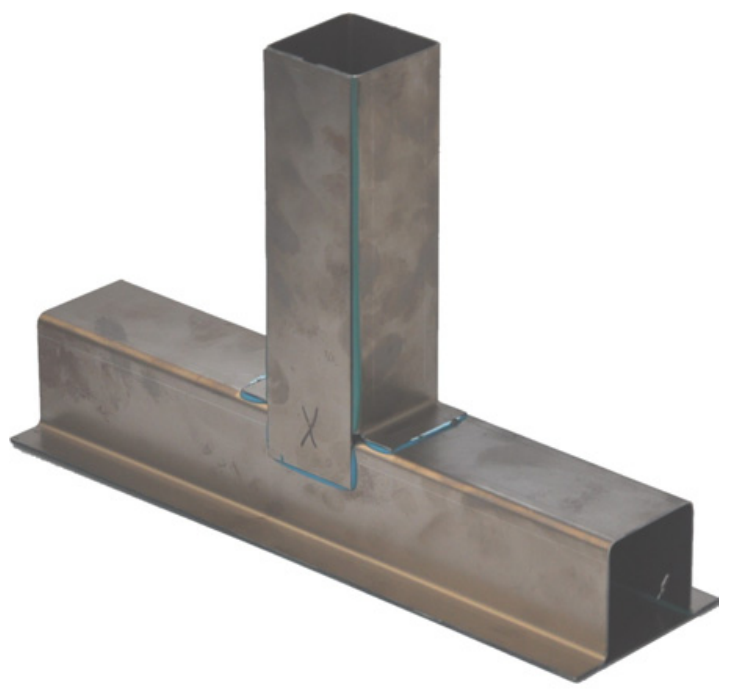

Fig. 1. New T-joint geometry.

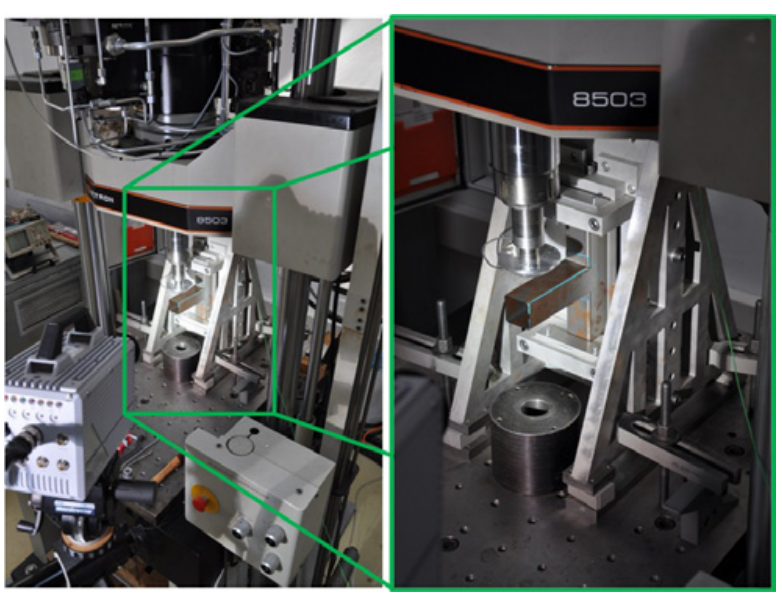

Fig. 2. Experimental setup.

which can be correlated to the initiation of peel failure at the edge of the upper adhesive bond lines. The force is then reduced slowly while the crack propagates along the length of the bond line. Complete decohesion of the adhesive bond line results in a significant load drop. Loads recorded after the major load drop are due to bending of the steel back face. A rate effect was observed. The load corresponding to damage initiation increase with increasing loading rate. For the highest loading rate, the force-disaplcement curve reproducibly feature a load drop followed by a load increase in the middle of the test. This is due to the impactor losing contact with the T-joint. For all loading rates, cohesive failure of the bond line was observed.

\subsubsection{Side impact}

High speed video footage of the typical failure sequence observed during the experiments is given in Fig. 5. Here, the damage and failure mechanisms observed are more complex than for the front impact. First, strong plastic deformation of the rectangular profile is observed (1). Then, two different mechanisms are initiated: cohesive damage
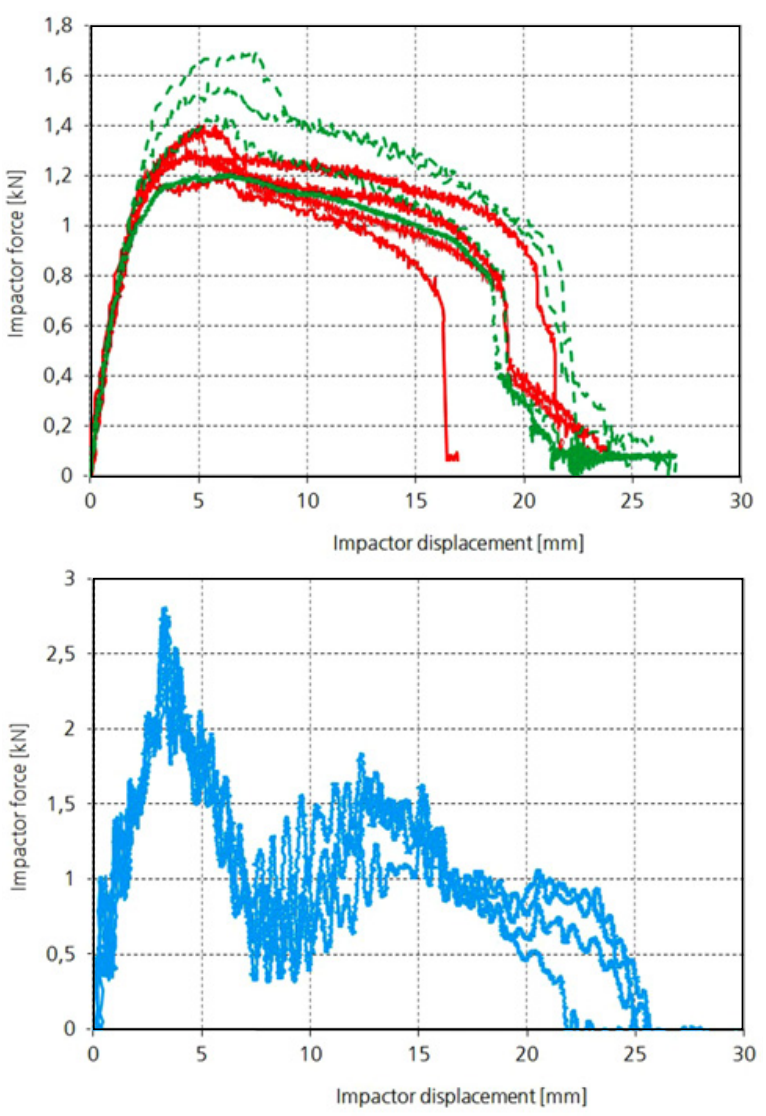

Fig. 3. Force-displacement curves recorded during front impact tests, the upper diagram shows quasi-static (red) and medium dynamic (green, dashed) results, the lower diagram shows crash loading.
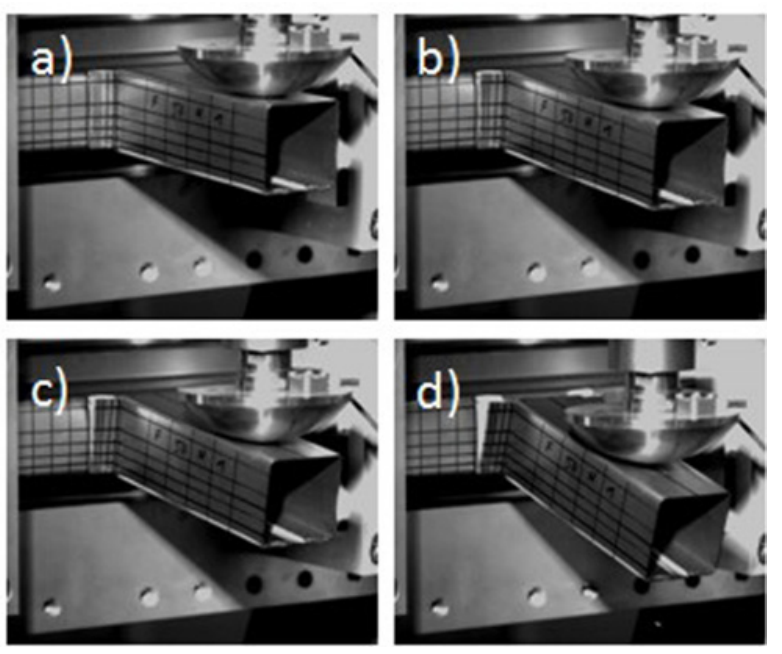

Fig. 4. Failure sequence during front impact.

at the upper adhesive bond line and plastic deformation of the back sheet resulting in the formation of a fold in the steel sheet (2). After complete decohesion of the upper bondline (3), the rectangular profile starts rotating resulting in even more pronounced folding accompanied by some slight debonding between the back face and the rectangular profile. Finally, shear failure of the adhesive bond line located at the side of the T-joint is observed (4). 


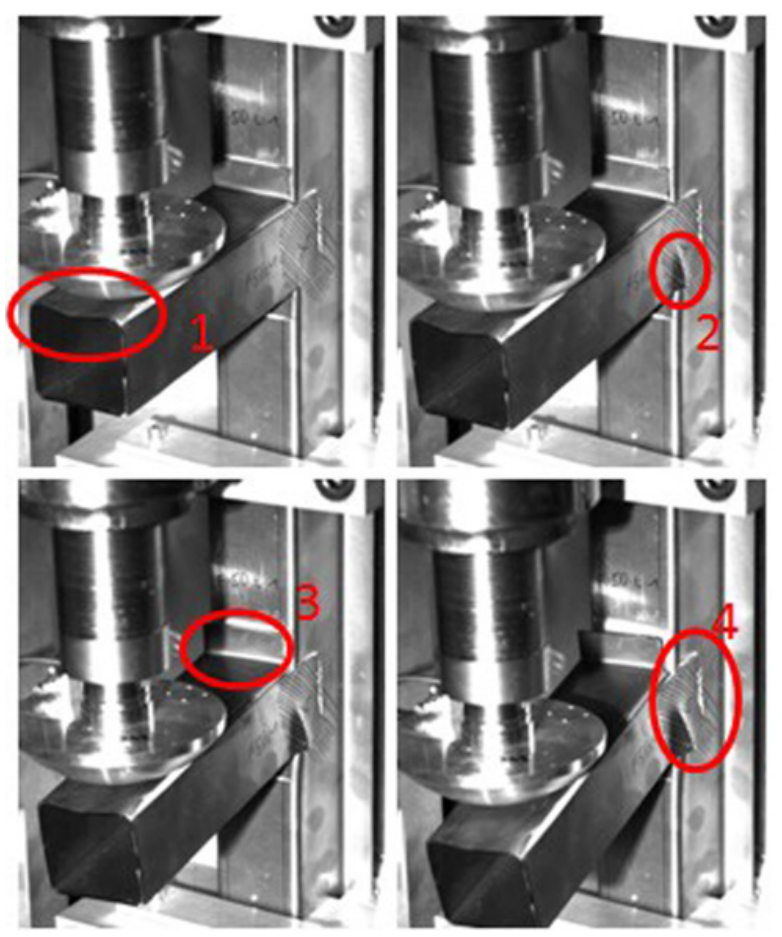

Fig. 5. Failure sequence during side impact.

The corresponding force-displacement responses are given in Fig. 6. The upper diagram compares quasi-static (red) and medium dynamic (green, dashed) results, the lower diagram shows force-displacement curves recorded during crash loading. Only a mild rate effect was observed regarding the peak load recorded during the test. However, a significant rate effect was observed regarding the failure mechanisms observed during the test and the displacement at final failure. For quasi-static loading, a combination of cohesive and adhesive failure was observed; for dynamic loading the failure mechanism was cohesive. This trend towards cohesive failure leads to a more pronounced fold formation (see Fig. 7). During this fold formation, the force remains at a plateau level of about $3.5 \mathrm{kN}$ until the adhesive bond line at the side of the T-joint fails under shear loading.

\section{Numerical analysis}

Quasi-static and dynamic Finite Element (FE) simulations were performed for the two different loading configurations described above using the commericial explicit FE code Abaqus/Explicit 6.11-1.

\subsection{Model setup}

The FE mesh for the T-joint model was created using Hypermesh. The steel sheets were modelled with shell elements, type S4R; the adhesive bond lines were modelled with cohesive interface elements, type COH3D8. The cohesive interface elements were connected to the shell elements using tied constraints. The element size was $5 \mathrm{~mm}$ for shell elements, which is typical for automotive crash simulation, the element size of the cohesive elements
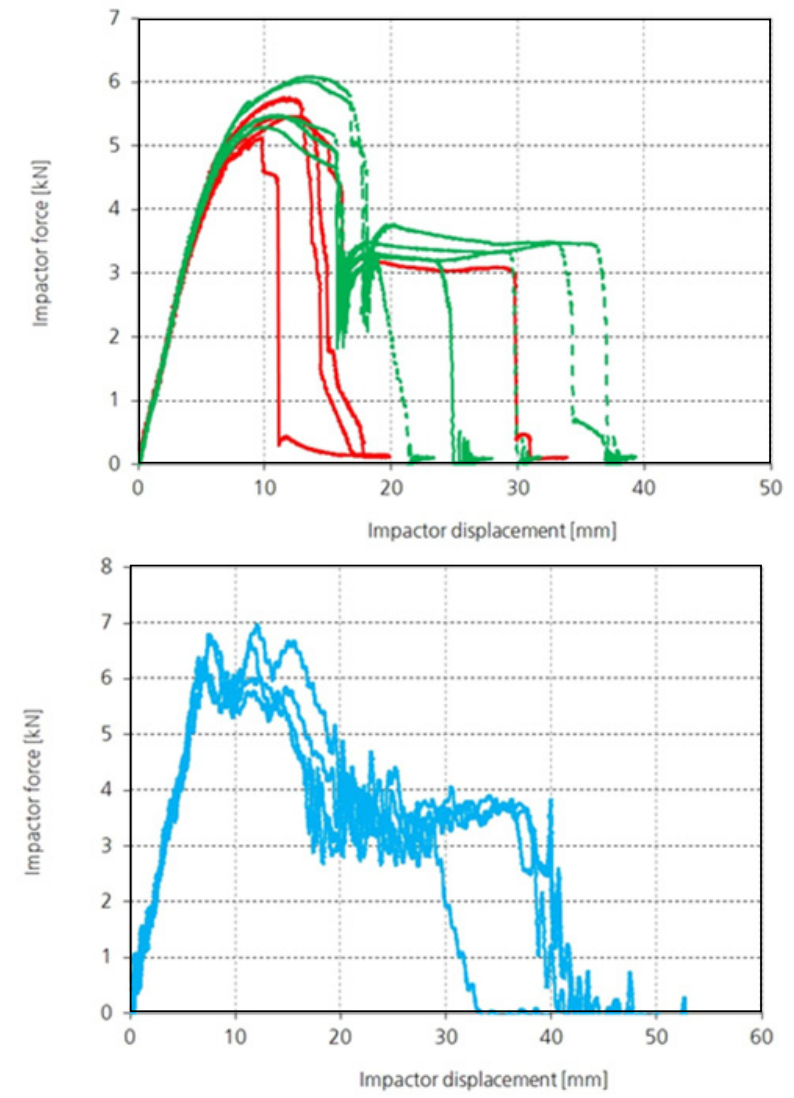

Fig. 6. Force-displacement curves recorded during side impact tests, the upper diagram shows quasi-static (red) and medium dynamic (green, dashed) results, the lower diagram shows crash loading.
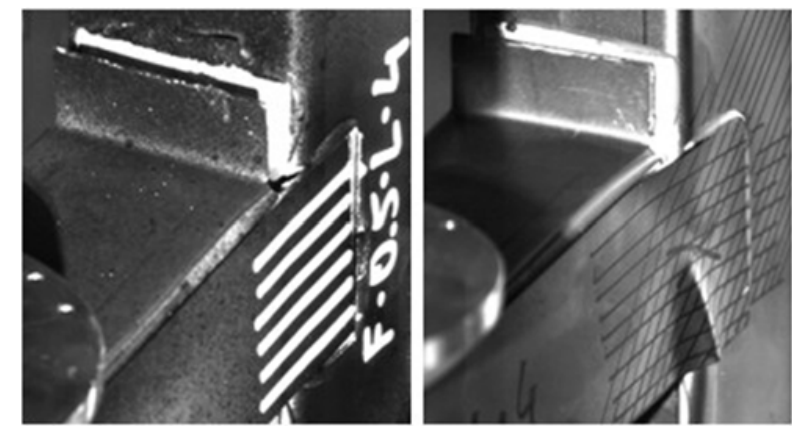

Fig. 7. Comparison of back sheet deformation just after bond line failure for quasi-static loading (left) and dynamic loading (right).

was $2 \mathrm{~mm}$. The shell elements was reduced to $2 \mathrm{~mm}$ in the regions where they connect to the cohesive elements. The aluminium impactor was modelled with solid elements, type C3D8R using a simple linear-elastic material model with elastic properties typical for aluminium. The steel sheets were modelled using an elastic-plastic material model considering isotropic hardening and rate sensitivity. This model was calibrated with tabular data available from tensile tests performed at nominal rates of $0.01 \mathrm{~s}^{-1}$, $1 \mathrm{~s}^{-1}$, and $200 \mathrm{~s}^{-1}$. A user-defined, fully rate dependent material model was chosen for the adhesive. The model consists of a stress based damage initiation criterion and an energy based failure criterion. The model combines 


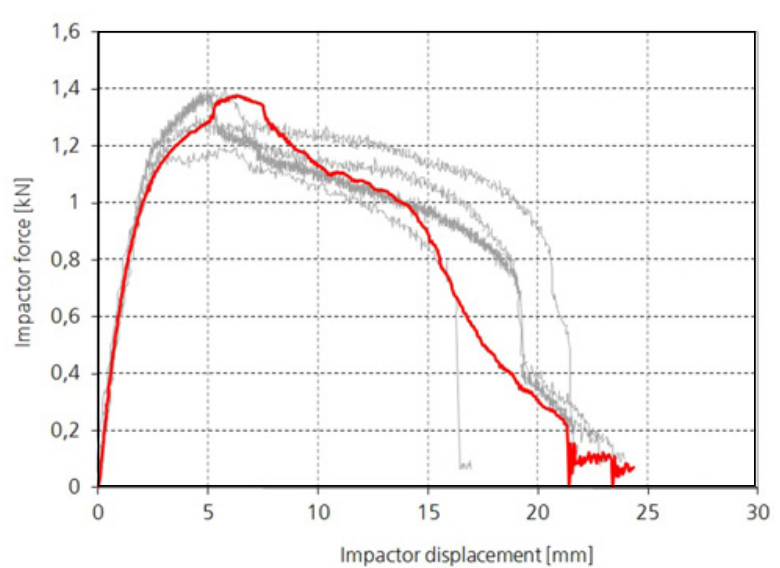

Fig. 8. Comparison of simulation and experiments for a front impact at $\mathrm{v}=0.5 \mathrm{~mm} / \mathrm{s}$.

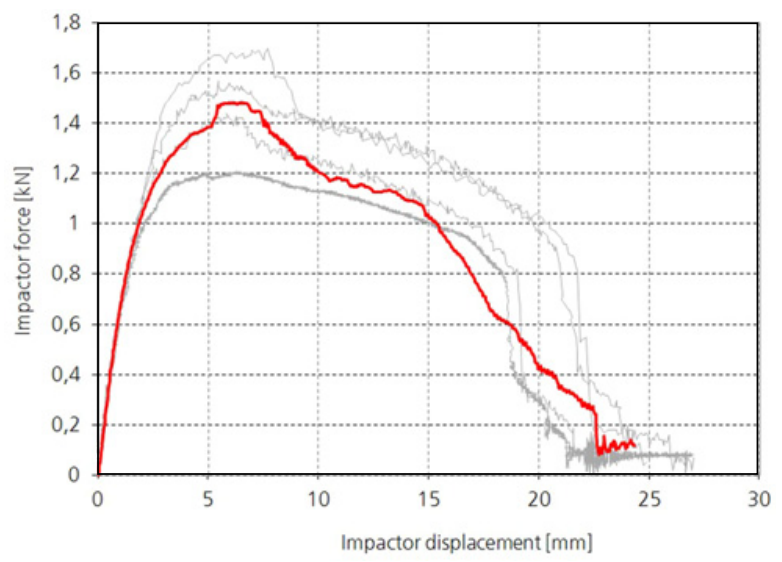

Fig. 9. Comparison of simulation and experiments for a front impact at $\mathrm{v}=50 \mathrm{~mm} / \mathrm{s}$.

a bi-linear traction-displacement curve for mode I (peel) loading with a tri-linear traction-displacement curve for mode II (shear) loading. Both, damage initiation stress and fracture toughness are rate dependent and are updated in each time step. More details can be found in [2]. Experimental data on the rate dependent behavior of mode I and mode II initiation stresses and fracture toughnesses, available in [1], was used for calibration of the adhesive model. General contact was applied to the whole model. The friction coefficients used in the contact formulation were found iteratively. A simplification was made for the representation of the clamping condition. Instead of modelling the whole rig including the clamps, it was shown to be sufficient to use fixed BC on a set of nodes at the location of the clamps. Selective mass scaling was used for the quasi-static and medium dynamic loading. Care was taken to ensure that mass scaling would not influence the simulation results.

\subsection{Results}

Figures 8 through 10 compare the numerical predictions for the T-joint subjected to front impact loading with the experimental data described in section 2.3.1. For all loading rates the predicted force-displacement curves show an

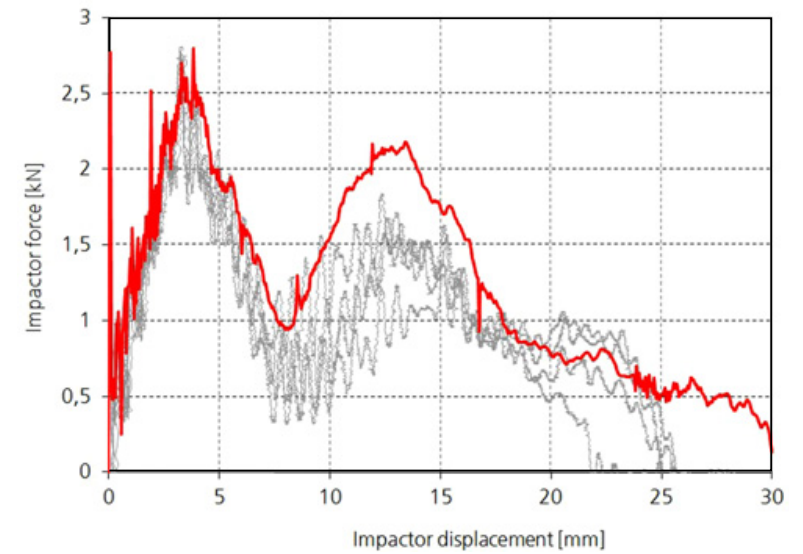

Fig. 10. Comparison of simulation and experiments for a front impact at $\mathrm{v}=4800 \mathrm{~mm} / \mathrm{s}$.

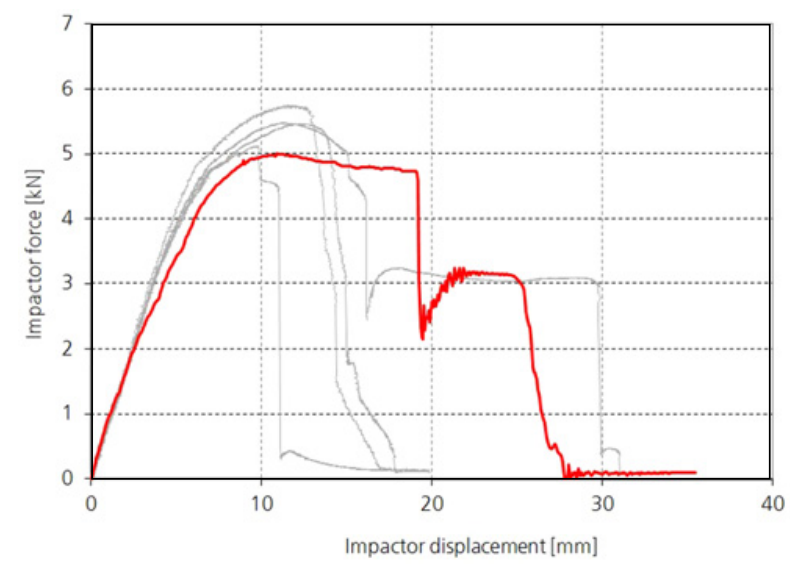

Fig. 11. Comparison of simulation and experiments for a side impact at $\mathrm{v}=0.5 \mathrm{~mm} / \mathrm{s}$.

excellent correlation with the experimental evidence. The rate dependent behavior is captured well. For all loading rates the predicted peak loads are within the experimental scatter. The predicted shapes of the load-displacement curves are captured reasonably well for the quasi-static and medium rate loading. Also the drastic change in shape for the highly dynamic loading is captured accurately. However, for an impactor displacement in the range of $15 \mathrm{~mm}$ to $20 \mathrm{~mm}$, the predicted damage evolution and the associated load reduction is overpredicted for quasi-static and medium rate loading. In [1] there was only limited data on the rate dependency of the fracture toughness which may be the reason for this deviation. The ongoing research project IGF $338 \mathrm{ZN}$ is investigating these rate effects in detail.

Figures 11 through 13 compare the numerical predictions for the T-joint subjected to side impact loading with the experimental data described in section 2.3.2. In the case of quasi-static loading, only one T-piece showed perfect cohesive failure. The corresponding force-displacement curve shows a significant plateau and a much higher displacement at failure than the experiments that exhibited a combined adhesive and cohesive failure mode. The simulation with cohesive interface elements cannot capture the undesired combination of adhesive and cohesive failure and can therefore only be compared against the data for the 


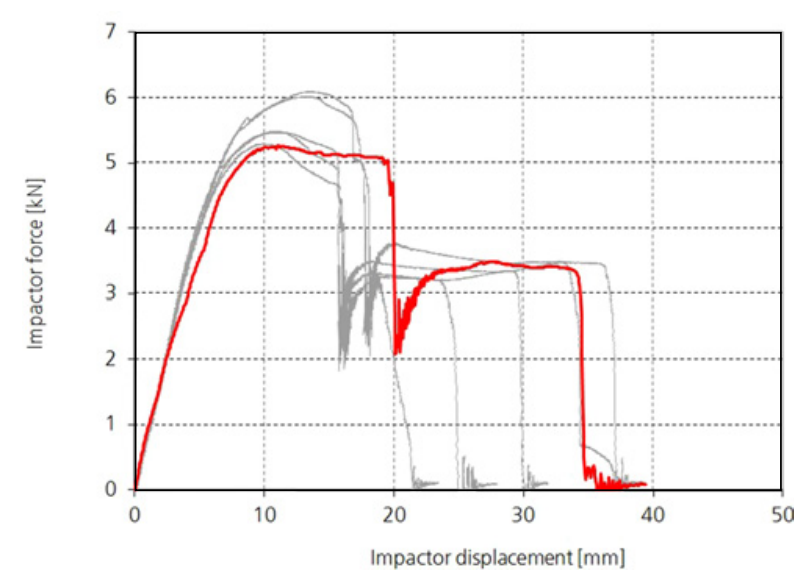

Fig. 12. Comparison of simulation and experiments for a side impact at $\mathrm{v}=50 \mathrm{~mm} / \mathrm{s}$.

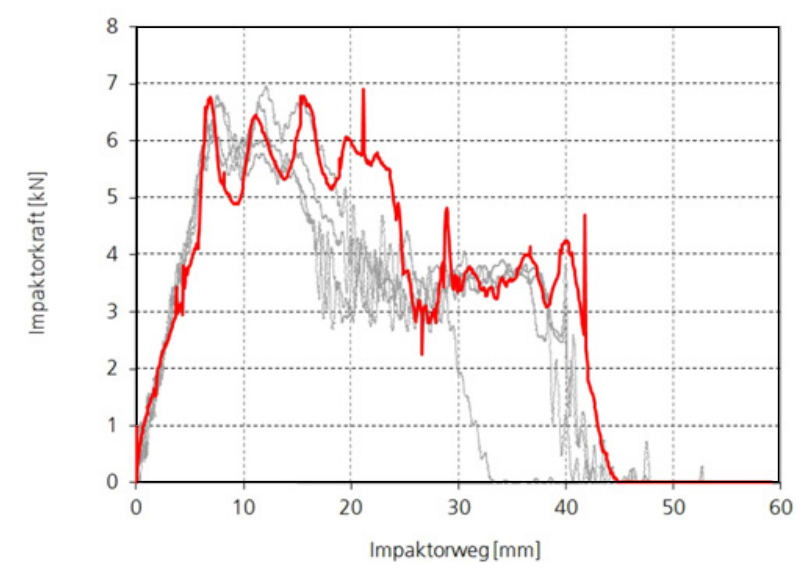

Fig. 13. Comparison of simulation and experiments for a side impact at $\mathrm{v}=4800 \mathrm{~mm} / \mathrm{s}$.

T-joint that showed cohesive failure. The simulation agrees reasonably well with the (limited) experimental data. For higher loading rates cohesive failure was observed in the experiments. The correlation between the predicted and the measured force-displacement curves is excellent. Also, all relevant damage and failure mechanisms oberved during the test such as slight debonding between the back sheet and the rectangular section or the fold formation in the back sheet were captured by the FE simulations as shown in Fig. 14. The amount of debonding between the back sheet and the rectangular profile predicted by dynamic simulations is bigger than for quasi-static simulations. The same trend was observed during the experiments. Also, the experimentally observed trend of a more pronounced fold formation with increasing loading rate was caputured by the simulations.

\section{Conclusions}

A new T-joint geometry was proposed allowing testing at different loading rates using an identical setup. Quasi-static and dynamic tests were performed in a front and a side impact configuration. Different failure mechanisms were triggered for different loading directions. Front impact loading of the T-joint caused peel failure of the upper bond lines. Side impact loading resulted in a complex

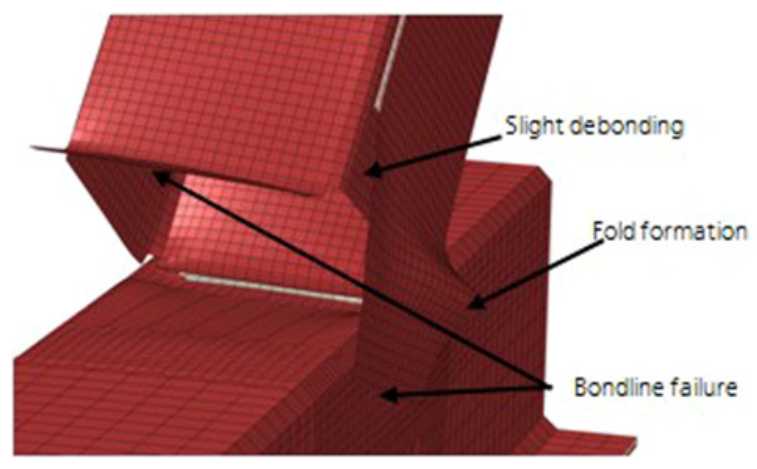

Fig. 14. Relevant damage and failure mechanisms captured in the side impact simulation.

combination of failure modes such as plastic deformation of the steel adherends, peel failure of the upper bond lines and shear failure of the bond line at the side of the lower profile. The experiments were highly reproducible except for quasi-static side impact loading where undesired combined adhesive and cohesive failure occurred during some tests. This test setup is therefore recommended for future analyses of adhesively bonded metallic joints.

A distinct rate effect was observed for experiments carried out in the front impact configuration. Here, an increase of loading rate caused an increase of measured peak load at damage initiation. Only a mild rate effect was observed for the side impact configuration.

The experiments were modelled using the explicit FE code Abaqus/Explicit. Rate dependent material models were used for the steel adherends and the adhesive. The FE predictions showed excellent correlations with the experimental evidence for all loading directions and loading rates.

\section{Acknowledgements}

This project was funded through the AiF research project "Robustheit und Zuverlaessigkeit der Berechnungsmethoden von Klebverbindungen mit hochfesten Stahlblechen unter Crashbelastungen", coordinated and financed by the "Forschungsvereinigung Stahlanwendung e.V.". The participants of this project are: Fraunhofer Institut für Kurzzeitdynamik (EMI) Freiburg, Fraunhofer Institut für Fertigungstechnik (IFAM) Bremen, Fraunhofer Institut für Werkstoffmechanik (IWM) Freiburg, Institut für Mechanik (IfM) Universität Kassel, and Lehrstuhl für technische Mechanik (LTM) Universität Paderborn. We are particularly grateful for the support given by Dr. Olaf Hesebeck (IFAM) and Dr. Stephan Marzi (IFAM) for manufacturing of the T-joints and many fruitful discussions.

\section{References}

1. M. Brede and F.-J. Heise (Eds.), Methodenentwicklung zur Berechnung von höherfesten Stahlklebverbindungen des Fahrzeugbaus unter Crashbelastung, Forschung für die Praxis P676, (2008)

2. M. May, M. Nossek, N. Petrinic, and S. Hiermaier, 3rd ECCOMAS Thematic Conference on the Mechanical Response of Composites, Hannover, (2011) 